\title{
EDITORIAL
}

\section{Congenital lifelong urology}

\author{
Dan Wood ${ }^{1} \cdot$ Hadley Wood ${ }^{2}$
}

Published online: 5 May 2021

(c) The Author(s), under exclusive licence to Springer-Verlag GmbH Germany, part of Springer Nature 2021

\section{Dear Editor}

This project is the culmination of a massive body of work. We are grateful to the International Consultation on Urological Diseases and the Société Internationale d'Urologie for supporting the first Worldwide Consultation, examining congenital lifelong urology [1]. We were privileged to work with an extraordinary group of authors and section editors, having created the original book they have kindly worked to produce these papers for the World Journal of Urology to whom we are equally grateful for supporting this special edition.

The necessity for care runs from cradle to grave for most patients born with congenital urological anomalies. Weiner et al. provide an overview of considerations from a pediatric perspective for patients born with conditions such as hypospadias, exstrophy, cloacal anomalies, posterior urethral valves and congenital neurogenic bladder. The basis of treatment is to preserve good urological function, including renal and bladder function, as well as optimize sexual and reproductive function. Part and parcel to this is education and prevention of secondary complications, including urinary tract infections and operative complications. The authors give an excellent overview of surgical techniques required in this practice and the importance of long-term follow-up. The range of conditions and potential sequelae necessitate engagement with a multidisciplinary team to provide optimal long-term care [2].

These themes are expanded by Peycelon and Misseri [3]. Their manuscript focuses on the critical period of development as a child moves through adolescence, into adulthood

\footnotetext{
Dan Wood

dan.wood1@nhs.net

Hadley Wood

WOODH@ccf.org

1 University College London Hospitals, London NW1 2BU, UK

2 Cleveland Clinic, Cleveland, OH 44195, USA
}

and achieving their independence in healthcare. We use the term "transition" to describe this process. During this period, puberty has the potential not just to impact independence and maturity, but also body mass, renal function and, of course, sexual and ultimately reproductive function. The potential for malignancy in some conditions, for example, previously undescended testes, is also addressed as surveillance become more relevant during young adulthood. Finally, while multidisciplinary care is important for many of these patients across the lifespan, it is particularly important during transition to ensure patients receive appropriate, uninterrupted care and are not lost to follow-up as they move into adulthood [3].

Yerkes et al. [4] takes a deeper dive into renal function, including measurement challenges and prevention of chronic kidney disease. For patients impacted by neurogenic bladder and vesicoureteric reflux, active monitoring and treatment is critical across the lifespan. Additionally, direct nephrotoxic insults, like the use of over the counter nephrotoxic medication or uncontrolled hypertension, can disproportionately impact patients with baseline renal insufficiency from congenintal causes. Stone management is particularly challenging in patients with complex congenital anatomy and many patients with congenital urological anomalies are at increased risk for both upper and lower tract stones. The ability of the team to anticipate and delay end stage renal failure is integral to the overall management of patients with early involvement of nephrology and timely referral to kidney transplant specialists [4].

Spinot and De Win [5, 6] focus specifically on sexual and reproductive function, highlighting the interaction between body image, the underlying condition alongside the discernable anatomical, surgical and hormonal challenges. Many adolescents and young adults are undereducated about sexual function and fertility, and this is particularly true for our population. While this education and knowledge gap is well described, many urologists and gynecologists feel illequipped to discuss or treat sexual dysfunction and infertility in patients with congenital conditions. For young women in this population, it is especially important to ensure that 
they understand how to protect their own health and what the ramifications may be for them and their offspring if and when they proceed with pregnancy. This includes education around pre-conception treatment (e.g., enhanced folic acid supplementation in patients with conditions such as spina bifida) as well as possible differences or health risks associated with pregnancy and delivery $[5,6]$.

Many patients with congenital bladder conditions undergo operations in childhood to facilitate storage and emptying of urine (e.g., augmentation cystoplasty). Nettey et al. have provided a comprehensive review of the long-term impact of these surgeries, including secondary operations that are almost always required in adult life. Bacteruria is normal for reconstructed and neurogenic bladders, this manuscript addresses how to identify, treat and prevent UTIs in this population. For patients performing self-catheterization to empty, new-onset incontinence or obstruction necessitates evaluation; Nettey et al. provide a framework for evaluation and treatment of problems that arise with Mitrofanoff channels and pouches in adult life [7].

As with all humans, patients with congenital urological anomalies are not spared age-related urological challenges. The final manuscript [8] examines how "normal" aging may affect those who have had treatment in childhood for a congenital urological condition. While basic guidelines for treatment of BPH or prostate cancer may be appropriate for most patients, special considerations should be made for patients with congenital neuropathic bladder prior to treatment. Perhaps, less well recognized but at least as important are the issues specific to this population around long-term fertility, hypogonadism, and sexual health.

We hope the readers find this resource useful in their own practices and that this issue contributes to overall improved urological care for this unique and heterogeneous population of patients.

\section{References}

1. Congenital lifelong urology: caring for the adolescent and adult patient with congenital and childhood gu conditions. (Société Internationale d'Urologie, 2018)

2. Wiener JS, Huck N, Blais A-S et al (2020) Challenges in pediatric urologic practice: a lifelong view. World J Urol. https://doi.org/10. 1007/s00345-020-03203-1

3. Peycelon M, Misseri R (2020) The basics of transition in congenital lifelong urology. World J Urol. https://doi.org/10.1007/ s00345-020-03116-z

4. Yerkes EB, Baum M, Chu DI (2020) Chronic kidney disease and upper tract concerns after congenital and acquired urinary tract abnormalities: considerations for transition of care in teens and young adults. World J Urol. https://doi.org/10.1007/ s00345-020-03273-1

5. De Win G, Dautricourt S, Deans R et al (2020) Fertility and sexuality issues in congenital lifelong urology patients: female aspects. World J Urol. https://doi.org/10.1007/s00345-020-03461-z

6. Spinoit AF, Waterschoot M, Sinatti C et al (2020) Fertility and sexuality issues in congenital lifelong urology patients: male aspects. World J Urol. https://doi.org/10.1007/s00345-020-03121-2

7. Nettey OS, Bowen DK, Santiago-Lastra Y et al (2020) Complications in adulthood for patients with paediatric genitourinary reconstruction. World J Urol. https://doi.org/10.1007/ s00345-020-03295-9

8. Faure Walker N, Gill B, Olsburgh J et al (2020) Age-related urologic problems in the complex urologic patient. World J Urol. https://doi.org/10.1007/s00345-020-03111-4

Publisher's Note Springer Nature remains neutral with regard to jurisdictional claims in published maps and institutional affiliations. 